\title{
Anti-tumor activity of metformin: from metabolic and epigenetic perspectives
}

\author{
Xilan Yu ${ }^{1, *}$, Wuxiang Mao ${ }^{1, *}$, Yansheng Zhai ${ }^{1}$, Chong Tong ${ }^{1}$, Min Liu ${ }^{1}$, Lixin Ma ${ }^{1}$, \\ Xiaolan $\mathbf{Y} \mathbf{u}^{1}$, Shanshan $\mathbf{L i}^{1}$ \\ ${ }^{1}$ Hubei Collaborative Innovation Center for Green Transformation of Bio-Resources, College of Life Sciences, Hubei University, \\ Wuhan, Hubei, China \\ *These authors have equally contributed to this work \\ Correspondence to: Xiaolan Yu, email: yxiaolan@163.com \\ Shanshan Li, email: shl@hubu.edu.cn
}

Keywords: metformin, therapeutic targets, metabolism, epigenetic modifications

Received: September 22, 2016

Accepted: November 03, 2016

Published: November 26, 2016

\begin{abstract}
Metformin has been used to treat type 2 diabetes for over 50 years. Epidemiological, preclinical and clinical studies suggest that metformin treatment reduces cancer incidence in diabetes patients. Due to its potential as an anti-cancer agent and its low cost, metformin has gained intense research interest. Its traditional anti-cancer mechanisms involve both indirect and direct insulin-dependent pathways. Here, we discussed the anti-tumor mechanism of metformin from the aspects of cell metabolism and epigenetic modifications. The effects of metformin on anti-cancer immunity and apoptosis were also described. Understanding these mechanisms will shed lights on application of metformin in clinical trials and development of anticancer therapy.
\end{abstract}

\section{INTRODUCTION}

Metformin is the world's widely prescribed agent for treatment of type 2 diabetes. Type 2 diabetes is a metabolic disease characterized by reduced tissue responsiveness to insulin, known as insulin resistance $[1,2]$. This leads to insufficient peripheral glucose elimination, impaired inhibition of hepatic gluconeogenesis and elevated hepatic glucose output. The anti-diabetic effect of metformin is primarily reflected in hepatocytes, myocytes, adipocytes, and $\beta$-pancreatic cells (Figure 1). Metformin inhibits glucose production in liver whereas increases insulin sensitivity in the peripheral tissues, resulting in elevated glucose uptake and consumption by skeletal muscle and adipose tissues (Figure 1) [1]. Metformin treatment reduces insulin secretion by $\beta$-pancreatic cells [2]. The key molecule that executes these functions is AMP-activated protein kinase (AMPK), a serine-threonine kinase regulating cellular energy metabolism (Figure 1). Activated AMPK impairs the overall synthesis of proteins, fatty acids, and cholesterol, but increases fatty acids usage [3].
Over the past decade, meta-analysis revealed that metformin-treated diabetic patients tend to have a reduced incidence of cancer [3]. The first evidence came from the report by Evans et al., who found an inverse relationship between metformin usage and cancer incidence for patients with type 2 diabetes [4]. Further studies with type 2 diabetes patients demonstrated that metformin reduced their risk of cancer at many sites, especially for breast, colorectal, ovarian and endometrial cancer [5]. The anti-cancer activity of metformin has also been shown for several cancers in animal models. Kisfalvi et al. reported that administration of metformin significantly reduced the growth of human pancreatic cancer cells xenografted on the flank of nude mice [6]. In addition, metformin significantly inhibits the colony formation and tumor growth in prostate, lung, or colon cancer cell xenografts [7-9]. Metformin has being used in clinical trials in cancer and is now extending to non-diabetic population $[5,10]$. In this review, we focused on discussing the potential mechanisms of metformin in cancer prevention and treatment. 


\section{CLASSIC ANTI-CANCER MECHANISMS OF METFORMIN}

\section{Direct or insulin-independent anti-cancer mechanisms of metformin}

The direct anti-cancer mechanism of metformin is activating the LBK1-AMPK signaling pathway (Figure 2). Metformin uncouples the electron transport chain at complex I, leading to impaired mitochondrial function, decreased adenosine triphosphate (ATP) synthesis, and elevated cellular AMP/ATP ratio [11]. Increased binding of AMP to AMPK activates AMPK by inducing phosphorylation of its catalytic subunit at residue Thr172 by liver kinase B1 (LKB1). LKB1 is a tumor suppressor and its mutations are associated with the PeutzJeghers cancer predisposition syndrome [12]. Binding of AMP to AMPK also prevents dephosphorylation of AMPK Thr172 by protein phosphatases. LKB1-activated AMPK phosphorylates and activates the tumor suppressor tuberous sclerosis complex 1 and 2 (TSC1/2), which negatively regulates the activity of mTOR by inhibiting Ras homolog enriched in brain (Rheb) [13]. mTOR is a critical mediator of the phosphatidylinositol-3-kinase/ protein kinase $\mathrm{B} / \mathrm{Akt}(\mathrm{PI} 3 \mathrm{~K} / \mathrm{PKB} / \mathrm{Akt}$ ) signaling pathway, which is one of the most frequently deregulated molecular networks in human cancer $[14,15]$. Metformin-activated AMPK inhibits mTOR and reduces the phosphorylation of its downstream targets, the eukaryotic initiation factor 4E-binding proteins (4EBPs) and ribosomal S6 kinases (S6Ks), leading to an inhibition of global protein synthesis, cell cycle progression, cell proliferation and angiogenesis $[2,5]$. Moreover, AMPK has been reported to suppress mTOR signaling pathway independent of TSC2 via phosphorylation of mTOR binding protein Raptor (Figure 2) [16].

Metformin also exerts anti-tumor effects independently of AMPK/LBK1/TSC signaling pathway. Metformin inhibits the mTOR signaling pathway by suppressing Rag GTPase-dependent activation of mTOR (Figure 2) [17]. Moreover, metformin inhibits mTOR independently of AMPK by up-regulating the expression of REDD1 to cause cell cycle arrest [18].

A recent study showed that in pancreatic cancer cells, metformin potentiates the anti-tumor activity of resveratrol by inhibiting vascular endothelial growth factor

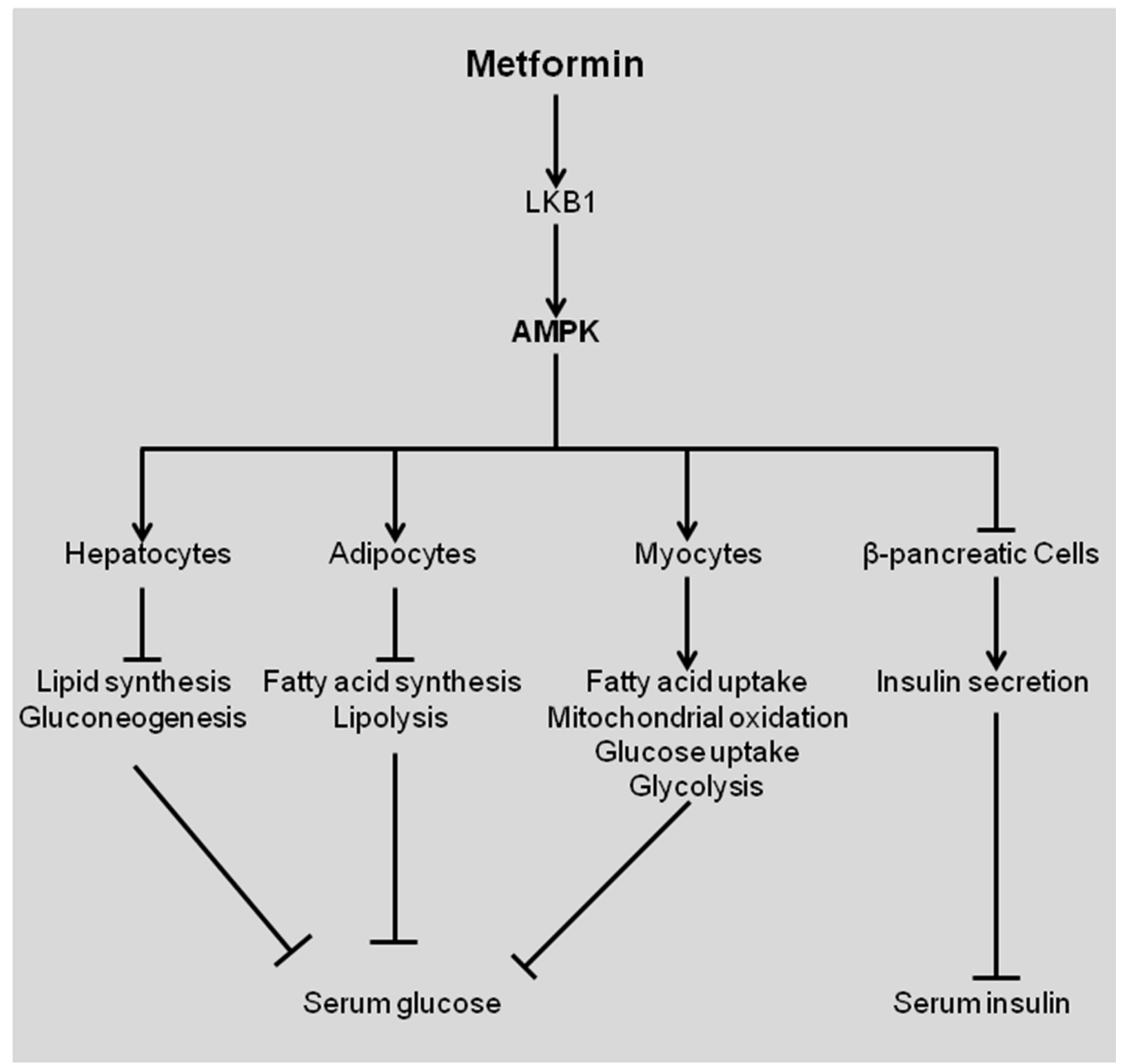

Figure 1: Effects of metformin in patients with diabetes type 2. Metformin activates LKB1, which then activates AMPK, resulting in differential effects in various tissues. Figures were adapted from [2]. AMPK, AMP-activated protein kinase. 
b (VEGF-B) signaling pathway [19]. Resveratrol has a dual role in pancreatic cancer cells: on one hand, it acts as a tumor suppressor by accelerating apoptosis; on the other hand, it promotes tumorigenesis by up-regulating the expression of VEGF-B, which then activates GSK-3 $\beta$ and inhibits apoptosis [20]. Metformin suppresses resveratrolinduced expression of VEGF-B, thereby potentiating the anti-cancer effect of resveratrol via inhibition of VEGF-B/ GSK-3 $\beta$ signaling pathway [19].

\section{Indirect or insulin-dependent anti-cancer mechanisms of metformin}

The indirect mechanism of metformin in anti-cancer function is related to its ability to lower insulin and insulinlike growth factor 1 (IGF-1) (Figure 3). Insulin increases glucose utilization, which facilitates tumor initiation and progression [5]. Exposure to elevated insulin increased the incidence of liver cancer and heptacellular carcinoma

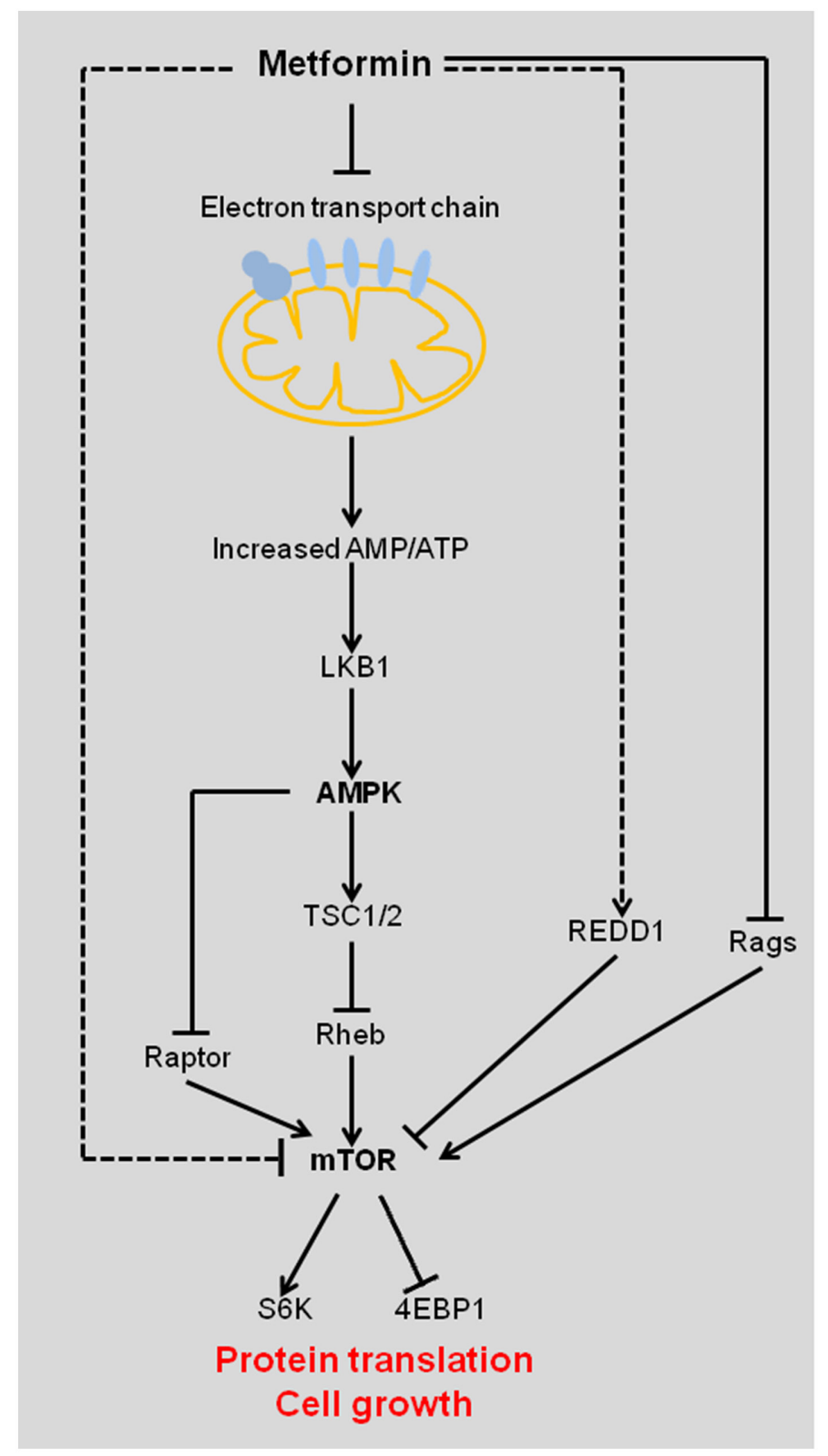

Figure 2: Diagram showing the indirect effect of metformin in suppressing tumorigenesis. Metformin inhibits complex I of the electron transport chain, which leads to increased AMP/ATP ratio and activation of AMPK by LKB1. Activated AMPK inhibits mTOR and its downstream targets by the following two pathways: 1. AMPK stabilizes TSC1/2, which inhibits Rheb, an activator of mTOR; 2 . AMPK inhibits mTOR binding protein Raptor. Metformin directly inhibits mTOR by up-regulating REDD1 and suppressing Rags. AMPK, AMP-activated protein kinase; Rheb, Ras homolog enriched in brain; LKB1, liver kinase B1; REDD1, regulated in development and DNA damage response 1; TSC, tuberous sclerosis complex; Rags, Rag GTPases; mTOR, mammalian target of rapamycin; 4EBP1, eukaryotic initiation factor 4E binding protein 1; S6K, S6 kinase. 
(HCC) [1]. IGF-1 is a more potent mitogen than insulin and, like insulin, binds to its IGF-binding protein (IGFBP) to stimulate cell growth and protect cells from apoptosis via Ras/Raf/MEK/ERK and PI3K/Akt/mTOR signaling pathways (Figure 3) [5]. IGF-1 also inhibits PTEN, which negatively regulates the $\mathrm{PI} 3 \mathrm{~K} / \mathrm{Akt} / \mathrm{mTOR}$ signaling pathway. Elevated insulin competes IGF-binding protein (IGFBP) with IGF-1, resulting in dissociation of IGF-1 from IGFBP to stimulate cell growth. Metformin reduces the overall insulin levels, which subsequently lowers free IGF-1 (Figure 3), thereby hindering cell growth [21]. The indirect mechanism of metformin in anti-cancer is primarily prosecuted in liver, where metformin inhibits hepatic gluconeogenesis [1].

\section{IMMUNE-MEDIATED ANTI-TUMOR EFFECT BY METFORMIN}

Metformin can exert anti-tumor activity by enhancing $\mathrm{CD}^{+} \mathrm{T}$ cells $[22,23] . \mathrm{CD}^{+} \mathrm{T}$ cells are key players in mediating immunity to tumors [24]. When stimulated with cancer specific antigen, $\mathrm{CD} 8^{+} \mathrm{T}$ cells initiate expansion and differentiation to effector cytotoxic $\mathrm{T}$ lymphocytes (CTLs). When antigen is cleared, most effector $\mathrm{CD}^{+} \mathrm{T}$ cells die followed by survival of long-lived memory cells [24]. Persistent stimulation by cancer cells makes $\mathrm{CD}^{+} \mathrm{T}$ cells lose the ability to secrete cytokines and undergo apoptosis, a process known as immune exhaustion. Pearce et al. reported that metformin increased $\mathrm{CD}^{+}$longlived memory cells to protect the mice vaccinated with attenuated Listeria monocytogenes expressing OVA from challenge by OVA-expressing tumor cells [23]. Eikawa et al. found that metformin can inhibit apoptosis of $\mathrm{CD}^{+}$tumor-infiltrating lymphocytes (TILs) and prevent immune exhaustion [22]. Metformin restored its multifunctionality of $\mathrm{CD}^{+}$TILs by shifting central memory T cells (TCM) to effector memory T cells (TEM), therefore conferring resistant to rechallenge with the same tumor cells [22].

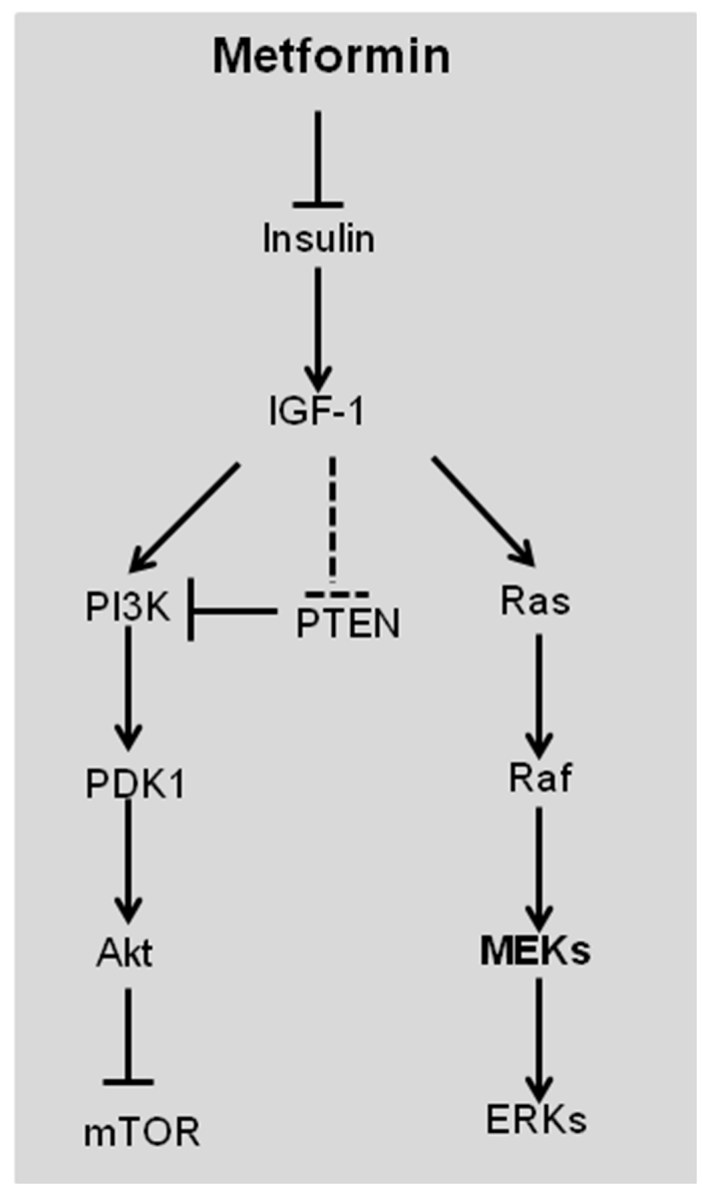

Figure 3: Schematic representation of the direct effect of metformin in suppressing tumorigenesis. Insulin increases free IGF-1 levels by displacing IGF-1 from its binding protein. IGF-1 stimulates tumorigenesis by Ras/Raf/MEK/ERK and PI3K/PDK1/Akt/ mTOR signal pathways. IGF-1 also negatively regulates PTEN, which inhibits PI3K/PDK1/Akt/mTOR signal pathway. Metformin reduces insulin and IGF-1 to inhibit cell proliferation and survival. IGF-1, insulin-like growth factor-1; PI3K, phosphoinositide 3-kinase; PDK1, phosphoinositide-dependent kinase 1; PTEN, phosphatase and tensin homolog. 


\section{IMPACT OF METFORMIN ON CELLULAR METABOLISM}

\section{Effect of metformin on glycolysis and tricarboxylic acid (TCA) cycle}

Cancer cells have distinct metabolism with normal cells. They have increased glycolysis but reduced oxidative phosphorylation, a phenomena known as "Warburg effect". The advantage of accelerated glycolysis in cancer cells is accumulation of sufficient amount of macromolecule intermediates that are critical for their survival and proliferation [25]. These macromolecules include ATP, nucleotides, lipids, as well as reduced nicotinamide adenine dinucleotide phosphate (NADPH) for macromolecular synthesis and redox homeostasis [26].

As a metabolism-control drug, one anti-cancer mechanism of metformin is interfering with cellular metabolism (Figure 4). The prominent change caused by metformin is increased glucose uptake, reduced accumulation of glycolytic intermediates at a specific stage of the pathway, and coordinately decreased TCA cycle intermediates including succinate, fumarate, malate, citrate, and $\alpha$-ketoglutarate $[27,28]$. Nonetheless, it should be noted that in breast cancer stem cells, biguanides only have a modest effect on glycolytic and TCA cycle intermediates, but they strongly deplete nucleotide triphosphates and may hinder nucleotide biosynthesis [28]. Hence, the effect of metformin on cancer metabolism differs depending on the cellular transformation stage [28].

\section{Effect of metformin on vitamin B12}

In addition to glycolysis and TCA cycle, metformin has been shown to reduce the cellular level of folate and vitamin B12 in patients with type 2 diabetes [29, 30]. This effect may enhance the anti-tumor efficacy of metformin, as vitamin B12 deficiency has been reported to inhibit tumor cells in vitro and increase tissue toxicity in patients receiving adjuvant chemotherapy $[2,31]$. Nonetheless, this mechanism deserves further attention in the clinical situation, since vitamin B12 deficiency has detrimental effects including neuropathy and anemia [2].

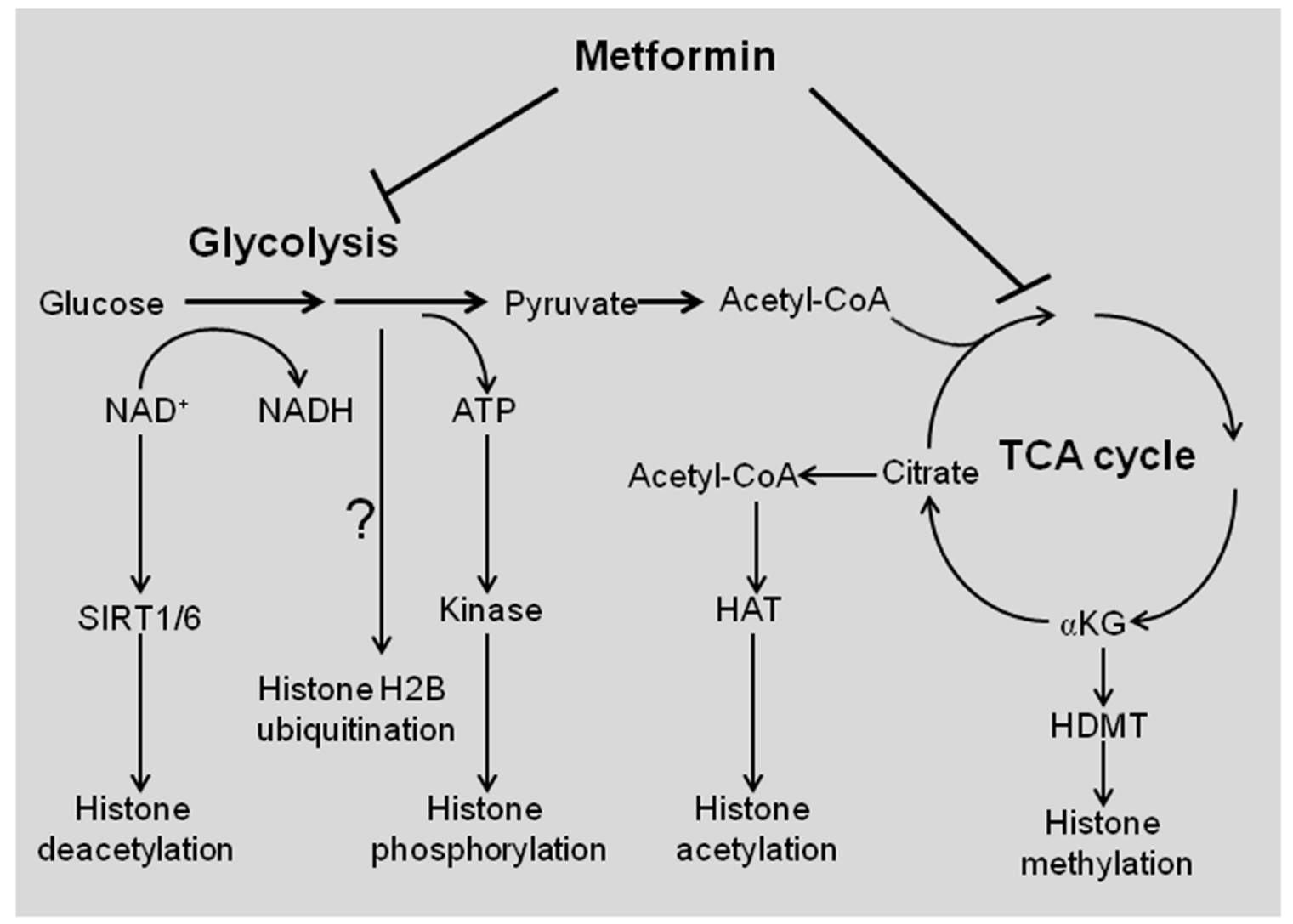

Figure 4: Schematic representation of metabolism-controlled histone modifications by metformin. Glycolysis determines the $\mathrm{NAD}^{+} / \mathrm{NADH}$ ratio, which enhances the activity of histone deacetylases (sirtuin) to reduce histone acetylation. Glycolysis provides ATP for protein kinase to phosphorylate histones. The TCA cycle intermediate citrate is converted to acetyl-CoA, which is used for HATmediated histone acetylation. Another TCA intermediate $\alpha \mathrm{KG}$ is used as cofactor to enhance the ability of HDMT to demethylate histones. Glycolysis is required for H2B ubiquitination, but it is unknown if metformin reduces H2B ubiquitination via blocking glycolysis. HAT, histone acetyltransferase; HDMT, histone demethylase; $\alpha \mathrm{KG}, \alpha$-ketoglutarate. 


\section{Is metformin equal to dietary restriction?}

Dietary restriction (DR) is the controlled reduction of food intake that can improve late-life health and increase lifespan. Several studies demonstrated that metformin treatment recapitulates the effects of DR, including improvement of late-life health and extension of lifespan in organisms ranging from nematodes to rodents and rhesus monkeys [32]. Extensive work done on animals indicate DR has an important role in suppressing certain cancer types [33]. It is uncertain whether metformin's antitumor properties are equal to DR.

\section{EFFECT OF METFORMIN ON EPIGENETIC MODIFICATIONS}

Cell metabolism is tightly linked to epigenetic modifications (Figure 4) [34, 35]. Cells adjust their metabolic states in response to extracellular signaling and/ or fuel availability by modulating their epigenetic program and gene transcription. The metabolic sensors that transduce the micro-environment changes to epigenetic modifications, are known as epigenetic "writers" or "erasers" [36].

The typical epigenetic modifications are histone modifications, including histone acetylation, methylation, phosphorylation, ubiquitination, etc. These modifications are catalyzed by specific histone modifying complexes, which require key metabolites as the acetyl, methyl, and phosphate donors (Figure 4). For example, chromatinmodifying enzymes consume key metabolites such as acetyl coenzyme A (acetyl-CoA) for acetylation, S-adenosylmethionine for methylation, and ATP for phosphorylation, etc. These enzymes include DNA methyltransferases (DNMTs), histone acetyltransferases (HATs), histone deacetylases (HDACs), histone methyltransferases (HMTs), and histone demethylases (HDMTs) [36]. Such substrates or cofactors are capable of diffusing through nuclear pores, therefore providing a rapid way to deliver metabolic information to nuclear functions. Thus, the cell's metabolic state could be reflected in the epigenetic modifications. In the following sections, we will discuss the impact of metformin on epigenetic modifications from histone and DNA modifications.

\section{Metformin and H2BK120 ubiquitination}

$\mathrm{Du}$ et al. reported that metformin reduced the global leves of histone H2B lysine 120 (H2BK120) monoubiquitination and the transcription of its target genes including p21 and cyclin D1, which are regulators of cell cycle [37]. However, it remains to be determined how metformin reduces H2BK120 ubiquitination and whether metformin down-regulates gene expression via H2BK120 ubiquitination. As metformin inhibits glycolysis [28] and glycolysis is required for H2B ubiquitination
[38], it is conceivable that metformin could reduce H2B ubiquitination by inhibiting glucose metabolism pathway. Further efforts are required to investigate this possibility.

\section{Metformin and histone acetyltransferases/ deacetylases}

Histone acetylation levels are determined by factors including histone acetyltransferases, histone deacetylases, and intracellular acetyl-CoA [39, 40]. Metformin-activated AMPK has been shown to enhance the activity of histone deacetylase SIRT1 to reduce acetylated p53 and expression of p21 [41]. However, in HepG2 cells, low concentration of metformin treatment suppresses the deacetylase activity of SIRT1 on p53, leading to increased p53 acetylation and cell senescence [10]. For histone acetylation, Khan et al. found that metformin inhibited diabetes-associated HDACs activity, thereby increased the acetylation of histone H3 in liver [42]. However, in breast cancer cells, metformin treatment reduced $\mathrm{H} 3 \mathrm{~K} 9$ acetylation [43]. It is unknown if metformin inhibits histone $\mathrm{H} 3$ acetylation by SIRT1. As metformin blocks glycolysis [28] and acetyl$\mathrm{CoA}$ is the downstream product of glycolysis, it will be interesting to investigate whether metformin reduces H3K9 acetylation via acetyl-CoA.

\section{Metformin and histone methyltransferase}

Transcriptome analysis showed that metformin down-regulated the expression of histone methyltransferase multiple myeloma SET domain (MMSET) in prostate cancer cells [44]. siRNA-mediated knockdown of MMSET attenuates cellular migration and invasion [44]. Hence, MMSET knockdown in combination with metformin treatment further reduced the capacity of prostate cancer cells to migrate and invade [44]. Banerjee et al. reported that metformin treatment increased global H3K4me2 but reduced the global levels of H3K27me2 and $\mathrm{H} 3 \mathrm{~K} 9 \mathrm{me} 2$ despite it is unknown how metformin alters these histone modifications and how the specificity is determined [43]. As metformin has been shown to decrease TCA cycle intermediate $\alpha$-ketoglutarate [27, 28], which is a co-activator of histone demethyhalses [45], it is possible that metformin affects histone methylation status via $\alpha$-ketoglutarate.

\section{Metformin and DNA methylation}

Yan et al. showed that metformin increased DNA methylation at the promoter of $\mathrm{H} 19$ [44], whose product is a long noncoding RNA implicated in the pathogenesis of diverse human cancers. H19 reduced the availability of let-7, a potent tumor suppressor microRNA that functions to suppress the expression of oncogenes important for cell growth and motility [44]. Metformin induced DNA methylation at $\mathrm{H} 19$ promoter to downregulate H19 expression and hence inhibit tumor cell 
migration and invasion [44]. Ishikawa et al. reported that metformin increased DNA methylation in insulin gene promoter, which reduced insulin gene transcription [46]. Much work is required for addressing which DNA methyltransferase(s) are responsible for DNA methylation changes and uncovering the underlying mechanisms.

\section{EFFECT OF METFORMIN ON APOPTOSIS}

Emerging evidence indicates that metformin induces apoptosis in several human cancers. Metformin has been shown to promote apoptosis by inducing the expression and/or activation of caspase 3 , the key molecule that executes apoptosis [47-49]. Gao et al. reported that metformin induced apoptosis by stimulating the production of reactive oxygen species (ROS) and reducing the mitochondrial membrane potential [50].

P53 is a tumor suppressor involved in DNA-damage repair and cell cycle control. AMPK can phosphorylate and activate p53, which then induces apoptosis of cells that encounter low nutrient conditions. Metformin could promote apoptosis by activating AMPK/p53 pathway [51]. Sun et al. showed that activated AMPK/p53 induces miR23a, which triggers apoptosis by inhibiting forkhead box protein A1 (FOXA1) in human hepatocellular carcinoma cells [52]. In addition, metformin triggers apoptosis by inducing metabolism reprogramming. Metformin blocks mitochondrial respiratory activity. As a compensatory response, cells need to enhance the p53-dependent fatty acid $\beta$-oxidation and glycolysis [8]. However, this metabolic conversion cannot be executed in p53-deficient cancer cells, leading to their apoptosis [8]. Metformin has also been found to reduce cell proliferation and migration in the oral squamous cell carcinoma (OSCC) cell lines probably by reversing Warburg effect [49]. In OSCC cells, metformin reduced the expression of hypoxia-inducible factor $1 \alpha(\mathrm{HIF}-1 \alpha)$ and increased pyruvate dehydrogenase (PDH) expression under hypoxic conditions [49]. PDH catalyzes the conversion of pyruvate to acetyl-CoA and hypoxic inhibition of PDH activity allows more pyruvate to be converted to lactate, which is necessary for cancer metabolic reprogramming and growth [53]. The upregulation of pyruvate dehydrogenase by metformin could promote cell apoptosis by attenuating the Warburg effect.

Other functions of metformin in anti-tumor activity include reducing c-myc protein [54], decreasing cell senescence [55], and inhibiting inflammatory response associated with cellular transformation [56]. Due to space limitations, we will not discuss the details here.

\section{CONCLUSIONS}

Metformin has been extensively studied to suppress tumorigenesis by direct and indirect mechanisms.
Recently, researchers began to elucidate the anti-tumor functions of metformin from the aspects of cellular metabolism and epigenetic modifications and much progress has been made. Still, a lot of questions remain to be dissolved. Firstly, there is no comprehensive study to investigate the impact of metformin on epigenetic modifications, such as histone modifications, DNA and/ or RNA methylation, noncoding RNA, etc. Secondly, it is unknown whether metformin affects chromatin structure, histone chaperones, etc. Lastly, how does metformin differentially regulate histone modifications, i.e. H3K4me2 versus H3K27me2? What are the key molecules that sense metformin and then transduce the signal to histone modifying enzymes?

The number of clinical trials with metformin in cancer continues to grow into the hundreds [5]. Most of these trials involve metformin in combination with other agents, implying that metformin alone is unlikely to give significant clinical benefits. Given the fact that metformin functions in cellular metabolism and epigenetic modifications, it is conceivable that a combination of metformin with drugs targeting these pathways will become promising anti-cancer therapy in the future. Indeed, Duo et al. showed that metformin can synergistically enhance the anti-tumor activity of histone deacetylase inhibitor trichostatin A against osteosarcoma cells [57]. The combination of metformin and the conventional chemotherapeutic agent, doxorubicin, kills both cancer stem cells and non-stem cancer cells in culture [58]. This combinatorial therapy effectively inhibits tumor growth in nude mice [58]. Clinical trials using a combination of metformin with traditional anticancer drugs have been performed in non-diabetic patients, especially in breast cancer patients [59, 60]. It is anticipated that much more efficient combined therapy will be developed with our understanding about the anticancer mechanism of metformin.

\section{ACKNOWLEDGMENTS}

We apologize to colleagues whose work cannot be cited here due to space limitation. We thank members of $\mathrm{Li}$ laboratory for critical reading of this manuscript.

\section{CONFLICTS OF INTEREST}

The authors declare that they have no conflicts of interest.

\section{GRANT SUPPORT}

This work was funded by grants from National Nature Science Foundation of China (No. 31671335 to Shanshan Li; No. 31600046 to Xilan Yu; No. 31672561 to Xiaolan $\mathrm{Yu}$ ). 


\section{REFERENCES}

1. Leone A, Di Gennaro E, Bruzzese F, Avallone A and Budillon A. New perspective for an old antidiabetic drug: metformin as anticancer agent. Cancer treatment and research. 2014; 159:355-376.

2. Jalving M, Gietema JA, Lefrandt JD, de Jong S, Reyners AK, Gans RO and de Vries EG. Metformin: taking away the candy for cancer? European journal of cancer. 2010; 46:2369-2380.

3. Dowling RJ, Goodwin PJ and Stambolic V. Understanding the benefit of metformin use in cancer treatment. BMC medicine. 2011; 9:33.

4. Evans JM, Donnelly LA, Emslie-Smith AM, Alessi DR and Morris AD. Metformin and reduced risk of cancer in diabetic patients. Bmj. 2005; 330:1304-1305.

5. Gong J, Kelekar G, Shen J, Shen J, Kaur S and Mita M. The expanding role of metformin in cancer: an update on antitumor mechanisms and clinical development. Targeted oncology. 2016; 11:447-467.

6. Kisfalvi K, Eibl G, Sinnett-Smith J and Rozengurt E. Metformin disrupts crosstalk between G protein-coupled receptor and insulin receptor signaling systems and inhibits pancreatic cancer growth. Cancer research. 2009; 69:6539-6545.

7. Ben Sahra I, Laurent K, Loubat A, Giorgetti-Peraldi S, Colosetti P, Auberger P, Tanti JF, Le Marchand-Brustel $\mathrm{Y}$ and Bost $\mathrm{F}$. The antidiabetic drug metformin exerts an antitumoral effect in vitro and in vivo through a decrease of cyclin D1 level. Oncogene. 2008; 27:3576-3586.

8. Buzzai M, Jones RG, Amaravadi RK, Lum JJ, DeBerardinis RJ, Zhao F, Viollet B and Thompson CB. Systemic treatment with the antidiabetic drug metformin selectively impairs p53-deficient tumor cell growth. Cancer research. 2007; 67:6745-6752.

9. Algire C, Zakikhani M, Blouin MJ, Shuai JH and Pollak M. Metformin attenuates the stimulatory effect of a highenergy diet on in vivo LLC1 carcinoma growth. Endocrinerelated cancer. 2008; 15:833-839.

10. Yi G, He Z, Zhou X, Xian L, Yuan T, Jia X, Hong J, He $\mathrm{L}$ and Liu J. Low concentration of metformin induces a p53-dependent senescence in hepatoma cells via activation of the AMPK pathway. International journal of oncology. 2013; 43:1503-1510.

11. El-Mir MY, Nogueira V, Fontaine E, Averet N, Rigoulet M and Leverve X. Dimethylbiguanide inhibits cell respiration via an indirect effect targeted on the respiratory chain complex I. The Journal of biological chemistry. 2000; 275:223-228.

12. Shaw RJ, Kosmatka M, Bardeesy N, Hurley RL, Witters LA, DePinho RA and Cantley LC. The tumor suppressor LKB1 kinase directly activates AMP-activated kinase and regulates apoptosis in response to energy stress. Proceedings of the National Academy of Sciences of the United States of America. 2004; 101:3329-3335.
13. Inoki $\mathrm{K}$, Zhu $\mathrm{T}$ and Guan KL. TSC2 mediates cellular energy response to control cell growth and survival. Cell. 2003; 115:577-590.

14. Lee JW, Soung YH, Kim SY, Lee HW, Park WS, Nam SW, Kim SH, Lee JY, Yoo NJ and Lee SH. PIK3CA gene is frequently mutated in breast carcinomas and hepatocellular carcinomas. Oncogene. 2005; 24:1477-1480.

15. Massacesi C, Di Tomaso E, Urban P, Germa C, Quadt C, Trandafir L, Aimone P, Fretault N, Dharan B, Tavorath R and Hirawat S. PI3K inhibitors as new cancer therapeutics: implications for clinical trial design. OncoTargets and therapy. 2016; 9:203-210.

16. Gwinn DM, Shackelford DB, Egan DF, Mihaylova MM, Mery A, Vasquez DS, Turk BE and Shaw RJ. AMPK phosphorylation of raptor mediates a metabolic checkpoint. Molecular cell. 2008; 30:214-226.

17. Kalender A, Selvaraj A, Kim SY, Gulati P, Brule S, Viollet B, Kemp BE, Bardeesy N, Dennis P, Schlager JJ, Marette A, Kozma SC and Thomas G. Metformin, independent of AMPK, inhibits mTORC1 in a rag GTPase-dependent manner. Cell metabolism. 2010; 11:390-401.

18. Ben Sahra I, Regazzetti C, Robert G, Laurent K, Le Marchand-Brustel Y, Auberger P, Tanti JF, Giorgetti-Peraldi $S$ and Bost F. Metformin, independent of AMPK, induces mTOR inhibition and cell-cycle arrest through REDD1. Cancer research. 2011; 71:4366-4372.

19. Zhu M, Zhang Q, Wang X, Kang L, Yang Y, Liu Y, Yang L, Li J, Yang L, Liu J, Li Y, Zu L, Shen Y and Qi Z. Metformin potentiates anti-tumor effect of resveratrol on pancreatic cancer by down-regulation of VEGF-B signaling pathway. Oncotarget. 2016; 7:84190-84200. doi: 10.18632/oncotarget.12391.

20. Yang L, Yang L, Tian W, Li J, Liu J, Zhu M, Zhang Y, Yang Y, Liu F, Zhang Q, Liu Q, Shen Y and Qi Z. Resveratrol plays dual roles in pancreatic cancer cells. Journal of cancer research and clinical oncology. 2014; 140:749-755.

21. Dowling RJ, Niraula S, Stambolic V and Goodwin PJ. Metformin in cancer: translational challenges. Journal of molecular endocrinology. 2012; 48:R31-43.

22. Eikawa S, Nishida M, Mizukami S, Yamazaki C, Nakayama $\mathrm{E}$ and Udono H. Immune-mediated antitumor effect by type 2 diabetes drug, metformin. Proceedings of the National Academy of Sciences of the United States of America. 2015; 112:1809-1814.

23. Pearce EL, Walsh MC, Cejas PJ, Harms GM, Shen H, Wang LS, Jones RG and Choi Y. Enhancing CD8 T-cell memory by modulating fatty acid metabolism. Nature. 2009; 460:103-107.

24. Prlic M, Williams MA and Bevan MJ. Requirements for CD8 T-cell priming, memory generation and maintenance. Current opinion in immunology. 2007; 19:315-319.

25. Vander Heiden MG, Cantley LC and Thompson CB. Understanding the Warburg effect: the metabolic requirements of cell proliferation. Science. 2009; 324:1029-1033. 
26. Amelio I, Cutruzzola F, Antonov A, Agostini M and Melino G. Serine and glycine metabolism in cancer. Trends in biochemical sciences. 2014; 39:191-198.

27. Zakikhani M, Bazile M, Hashemi S, Javeshghani S, Avizonis D, St Pierre J and Pollak MN. Alterations in cellular energy metabolism associated with the antiproliferative effects of the ATM inhibitor KU-55933 and with metformin. PloS one. 2012; 7:e49513.

28. Janzer A, German NJ, Gonzalez-Herrera KN, Asara JM, Haigis MC and Struhl K. Metformin and phenformin deplete tricarboxylic acid cycle and glycolytic intermediates during cell transformation and NTPs in cancer stem cells. Proceedings of the National Academy of Sciences of the United States of America. 2014; 111:10574-10579.

29. Akinlade KS, Agbebaku SO, Rahamon SK and Balogun WO. Vitamin B12 Levels In Patients with Type 2 Diabetes Mellitus on Metformin. Annals of Ibadan postgraduate medicine. 2015; 13:79-83.

30. Fogelman Y, Kitai E, Blumberg G, Golan-Cohen A, Rapoport $\mathrm{M}$ and Carmeli E. Vitamin B12 screening in metformin-treated diabetics in primary care: were elderly patients less likely to be tested? Aging clinical and experimental research. 2016; doi: 10.1007/ s40520-016-0546-1.

31. Garcia A and Tisman G. Metformin, B(12), and enhanced breast cancer response to chemotherapy. Journal of clinical oncology. 2010; 28:e19; author reply e20.

32. Cabreiro F, Au C, Leung KY, Vergara-Irigaray N, Cocheme HM, Noori T, Weinkove D, Schuster E, Greene ND and Gems D. Metformin retards aging in C. elegans by altering microbial folate and methionine metabolism. Cell. 2013; 153:228-239.

33. Astagimath MN and Rao SB. Dietary restriction (DR) and its advantages. Indian journal of clinical biochemistry. 2004; 19:1-5.

34. Li S, Swanson SK, Gogol M, Florens L, Washburn MP, Workman JL and Suganuma T. Serine and SAM Responsive Complex SESAME Regulates Histone Modification Crosstalk by Sensing Cellular Metabolism. Molecular cell. 2015; 60:408-421.

35. Yu X and Li S. Non-metabolic functions of glycolytic enzymes in tumorigenesis. Oncogene. 2016; doi: 10.1038/ onc. 2016.410

36. Li S, Kong L, Yu X and Zheng Y. Host-virus interactions: from the perspectives of epigenetics. Reviews in medical virology. 2014; 24:223-241.

37. Du Y, Zheng $\mathrm{H}$, Wang J, Ren Y, Li M, Gong C, $\mathrm{Xu} \mathrm{F}$ and Yang C. Metformin inhibits histone H2B monoubiquitination and downstream gene transcription in human breast cancer cells. Oncology letters. 2014; 8:809-812.

38. Gao $\mathrm{Z}$ and $\mathrm{Xu} \mathrm{CW}$. Glucose metabolism induces monoubiquitination of histone $\mathrm{H} 2 \mathrm{~B}$ in mammalian cells.
Biochemical and biophysical research communications. 2011; 404:428-433.

39. Li S and Shogren-Knaak MA. Cross-talk between histone H3 tails produces cooperative nucleosome acetylation. Proceedings of the National Academy of Sciences of the United States of America. 2008; 105:18243-18248.

40. Li S and Shogren-Knaak MA. The Gen5 bromodomain of the SAGA complex facilitates cooperative and crosstail acetylation of nucleosomes. The Journal of biological chemistry. 2009; 284:9411-9417.

41. Zhang E, Guo Q, Gao H, Xu R, Teng S and Wu Y. Metformin and Resveratrol Inhibited High GlucoseInduced Metabolic Memory of Endothelial Senescence through SIRT1/p300/p53/p21 Pathway. PloS one. 2015; 10:e0143814.

42. Khan S and Jena G. Sodium butyrate reduces insulinresistance, fat accumulation and dyslipidemia in type-2 diabetic rat: A comparative study with metformin. Chemicobiological interactions. 2016; 254:124-134.

43. Banerjee P, Surendran H, Chowdhury DR, Prabhakar K and Pal R. Metformin mediated reversal of epithelial to mesenchymal transition is triggered by epigenetic changes in E-cadherin promoter. Journal of molecular medicine. 2016.

44. Yan L, Zhou J, Gao Y, Ghazal S, Lu L, Bellone S, Yang Y, Liu N, Zhao X, Santin AD, Taylor H and Huang Y. Regulation of tumor cell migration and invasion by the H19/let-7 axis is antagonized by metformin-induced DNA methylation. Oncogene. 2015; 34:3076-3084.

45. Boukouris AE, Zervopoulos SD and Michelakis ED. Metabolic Enzymes Moonlighting in the Nucleus: Metabolic Regulation of Gene Transcription. Trends in biochemical sciences. 2016.

46. Ishikawa $\mathrm{K}$, Tsunekawa $\mathrm{S}$, Ikeniwa $\mathrm{M}$, Izumoto $\mathrm{T}$, Iida A, Ogata H, Uenishi E, Seino Y, Ozaki N, Sugimura Y, Hamada Y, Kuroda A, Shinjo K, Kondo Y and Oiso Y. Long-term pancreatic beta cell exposure to high levels of glucose but not palmitate induces DNA methylation within the insulin gene promoter and represses transcriptional activity. PloS one. 2015; 10:e115350.

47. Janjetovic K, Harhaji-Trajkovic L, Misirkic-Marjanovic M, Vucicevic L, Stevanovic D, Zogovic N, SumaracDumanovic M, Micic D and Trajkovic V. In vitro and in vivo anti-melanoma action of metformin. European journal of pharmacology. 2011; 668:373-382.

48. Cantrell LA, Zhou C, Mendivil A, Malloy KM, Gehrig PA and Bae-Jump VL. Metformin is a potent inhibitor of endometrial cancer cell proliferation--implications for a novel treatment strategy. Gynecologic oncology. 2010; 116:92-98.

49. Guimaraes TA, Farias LC, Santos ES, de Carvalho Fraga CA, Orsini LA, de Freitas Teles L, Feltenberger JD, de Jesus SF, de Souza MG, Santos SH, de Paula AM, Gomez RS and Guimaraes AL. Metformin increases PDH and suppresses 
HIF-1alpha under hypoxic conditions and induces cell death in oral squamous cell carcinoma. Oncotarget. 2016; 7:55057-55068. doi: 10.18632/oncotarget.10842.

50. Gao ZY, Liu Z, Bi MH, Zhang JJ, Han ZQ, Han X, Wang HY, Sun GP and Liu H. Metformin induces apoptosis via a mitochondria-mediated pathway in human breast cancer cells in vitro. Experimental and therapeutic medicine. 2016; 11:1700-1706.

51. Li P, Zhao M, Parris AB, Feng $X$ and Yang $X$. p53 is required for metformin-induced growth inhibition, senescence and apoptosis in breast cancer cells. Biochemical and biophysical research communications. 2015; 464:1267-1274.

52. Sun Y, Tao C, Huang X, He H, Shi H, Zhang Q and Wu $\mathrm{H}$. Metformin induces apoptosis of human hepatocellular carcinoma HepG2 cells by activating an AMPK/p53/miR23a/FOXA1 pathway. OncoTargets and therapy. 2016; 9:2845-2853.

53. Golias T, Papandreou I, Sun R, Kumar B, Brown NV, Swanson BJ, Pai R, Jaitin D, Le QT, Teknos TN and Denko NC. Hypoxic repression of pyruvate dehydrogenase activity is necessary for metabolic reprogramming and growth of model tumours. Scientific reports. 2016; $6: 31146$.

54. Akinyeke T, Matsumura S, Wang X, Wu Y, Schalfer ED, Saxena A, Yan W, Logan SK and Li X. Metformin targets c-MYC oncogene to prevent prostate cancer. Carcinogenesis. 2013; 34:2823-2832.

55. Noren Hooten N, Martin-Montalvo A, Dluzen DF, Zhang Y, Bernier M, Zonderman AB, Becker KG, Gorospe M, de Cabo R and Evans MK. Metformin-mediated increase in DICER1 regulates microRNA expression and cellular senescence. Aging cell. 2016; 15:572-581.

56. Hirsch HA, Iliopoulos D and Struhl K. Metformin inhibits the inflammatory response associated with cellular transformation and cancer stem cell growth. Proceedings of the National Academy of Sciences of the United States of America. 2013; 110:972-977.

57. Duo J, Ma Y, Wang G, Han X and Zhang C. Metformin synergistically enhances antitumor activity of histone deacetylase inhibitor trichostatin a against osteosarcoma cell line. DNA and cell biology. 2013; 32:156-164.

58. Hirsch HA, Iliopoulos D, Tsichlis PN and Struhl K. Metformin selectively targets cancer stem cells, and acts together with chemotherapy to block tumor growth and prolong remission. Cancer research. 2009; 69:7507-7511.

59. Hadad SM, Coates P, Jordan LB, Dowling RJ, Chang MC, Done SJ, Purdie CA, Goodwin PJ, Stambolic V, MoulderThompson S and Thompson AM. Evidence for biological effects of metformin in operable breast cancer: biomarker analysis in a pre-operative window of opportunity randomized trial. Breast cancer research and treatment. 2015; 150:149-155.

60. Martin-Castillo B, Dorca J, Vazquez-Martin A, OliverasFerraros C, Lopez-Bonet E, Garcia M, Del Barco S and Menendez JA. Incorporating the antidiabetic drug metformin in HER2-positive breast cancer treated with neo-adjuvant chemotherapy and trastuzumab: an ongoing clinical-translational research experience at the Catalan Institute of Oncology. Annals of oncology. 2010; 21:187-189. 